\title{
Induction of apoptosis by chitosan/HPV16 E7 siRNA complexes in cervical cancer cells
}

\author{
JUN YANG $^{1}$, SHAOPING LI ${ }^{1}$, FANG GUO ${ }^{1}$, WEI ZHANG ${ }^{1}$, YONGLIAN WANG ${ }^{1}$ and YING PAN $^{2}$ \\ ${ }^{1}$ Department of Obstetrics and Gynecology, The First Affiliated Hospital of Xinxiang Medical College, Henan 453100; \\ ${ }^{2}$ Department of Obstetrics and Gynecology, The Third Affiliated Hospital of Xinxiang Medical College, \\ Henan 453003, P.R. China
}

Received September 8, 2012; Accepted December 6, 2012

DOI: $10.3892 / \mathrm{mmr} .2012 .1246$

\begin{abstract}
Consecutive expression of the high-risk human papillomavirus (HPV) oncoproteins, E6 and E7, is pivotal for malignant transformation and maintenance of the malignant phenotype. These oncogenes may be potential targets of gene silencing-based molecular therapies for human cervical cancer. The aim of the present study was to evaluate the efficacy of chitosan-based HPV16 E7 siRNA delivery and the chitosan/HPV16 E7 siRNA complex in the induction of apoptosis in CaSki cells constitutively expressing HPV16 E6 and E7. Chitosan/siRNA nanoparticles were prepared by adding a chitosan solution drop-wise to an equal volume of siRNA solution. Formation of the chitosan/siRNA complex was verified by gel retardation assays and the entry of siRNA into the cells was confirmed by fluorescence microscopy. Expression of HPV16 E7 was examined by western blot analysis and apoptotic cells were detected by TUNEL staining. Chitosan formed complexes with HPV16 E7 siRNA. The chitosan/siRNA nanoparticles were efficiently delivered into CaSki cells and were observed to induce apoptosis. In conclusion, chitosan is suitable for use as a carrier for delivery of siRNA into cancer cells. The delivery of chitosan/HPV16 E7 siRNA nanoparticles in vivo may serve as a promising therapy for cervical cancer.
\end{abstract}

\section{Introduction}

Cervical cancer is a malignant tumor and the second most malignant cancer in females. It is a major threat to female health worldwide. Globally, 500,000 new cases and >250,000 mortalities occur each year. These figures account for $\sim 5 \%$ of all cancer cases worldwide with $\sim 80 \%$ of new cases reported in developing countries (1). In China, the annual incidence

Correspondence to: Dr Ying Pan, Department of Obstetrics and Gynecology, The Third Affiliated Hospital of Xinxiang Medical University, Wuyi Road, Xinxiang, Henan 453003, P.R. China

E-mail: yingpancn@126.com

Key words: chitosan, siRNA, nanoparticles, cervical cancer, apoptosis of new cervical cancer cases exceeds 130,000, accounting for $28.8 \%$ of new cases worldwide (2). An estimated 20,000 individuals succumb to cervical cancer every year in China, and incidence is increasing in young adults (3).

The most important risk factor for cervical cancer is infection with human papilloma virus (HPV), which accounts for $50-70 \%$ of all cervical cancer cases worldwide. Oncoproteins encoded by two early HPV genes, E6 and E7, are important for cell cycle control. E6 and E7 are required for malignant transformation and maintenance of malignant phenotypes and are crucial for the development and progression of cervical cancer (4-8). HPV16 E7 binds to key tumor suppressors and inhibits their activity. One of the most important targets of HPV16 E7 is the retinoblastoma protein (pRb) family which contains pRb, p107 and p130. In normal cells, pRb proteins are major regulators of the cell cycle, binding directly to the E2F transcription factor and negatively regulating its activity, thus inhibiting expression of E2F target genes important for cell cycle progression (9-16). In HPV16 E7-overexpressing cells, HPV16 E7 binds to pRb via its CR3 region. This binding induces $\mathrm{pRb}$ degradation through the ubiquitin-proteasome system and releases E2F into the cytosol (17-20). The free E2F translocates to the nucleus, activates the transcription of its target genes and promotes cell transformation. Therefore, suppression of HPV16 E7 expression is likely to inhibit cell growth and induce apoptosis and senescence, which may limit the growth of cancer cells.

RNA interference (RNAi) has become widely used as an experimental tool to analyze gene function and holds great promise in the field of gene therapy in cancer. However, several limitations restrict its use in basic research and clinical application. First, siRNA is not stable and is easily degradated by enzymes. Second, the delivery of siRNA into cells is a great challenge. Although liposome and cationic polymers have been used as carriers for siRNA delivery, these reagents are toxic to cells and not suitable for in vivo transfection. Chitosan is derived from chitin, the most abundant biopolymer in nature following cellulose and is a biologically safe, non-toxic, biodegradable and biocompatible polymer. It contains abundant - $\mathrm{NH} 2$ groups and is therefore positively charged at specific $\mathrm{pH}$ levels, enabling it to complex with negatively charged nanoparticles $(21,22)$. In the present study, chitosan was utilized as a carrier for delivery of HPV16 E7 siRNA into CaSki cells constitutively expressing 
HPV16 E6 and E7. The effect of chitosan/siRNA nanoparticles on the induction of apoptosis in these cells was examined. Results indicate a potential use of chitosan/siRNA complexes in the treatment of diseases, including cervical cancer.

\section{Materials and methods}

Materials. Chitosan was purchased from Jinan Haidebei Marine Bioengineering Co., Ltd. (Jinan, China). The degree of deacetylation was $86 \%$. The following siRNA oligos for HPV16 E7 were used: sense, GCATGGAGATACACCTACA and antisense, TGTAGGTGTATCTCCATGC (synthesized by Shanghai Generay Biotech Co., Ltd., Shanghai, China). The study was approved by the ethics committee of the Third Affiliated Hospital of Xinxiang Medical University, Xinxiang, Henan Province, China.

Preparation and characterization of chitosan/siRNA nanoparticles. Chitosan was dissolved in aqueous acetic acid (0.1 M sodium acetate/0.1 M acetic acid, $\mathrm{pH} 4.5)$ to prepare various concentrations of chitosan solution $(25-300 \mu \mathrm{g} / \mathrm{ml})$. Chitosan/siRNA nanoparticles were prepared by adding a chitosan solution drop-wise to an equal volume of siRNA solution $(20 \mu \mathrm{g} / \mathrm{ml})$ and incubating at room temperature for $30 \mathrm{~min}$. The chitosan was complexed with siRNA at a weight ratio of 1.25:1-15:1. The size and $\zeta$ potential of nanoparticles were measured using the submicron particle analysis system 4700 (Beckman Coulter Inc., Miami, FL, USA) and the Zetasizer Nano S (Malvern Instruments, Malvern, UK), respectively.

Measurement of siRNA loading efficiency. Chitosan and siRNA were mixed and the mixture was centrifuged and the absorbance of supernatant was measured at $260 \mathrm{~nm}$ to determine the concentration of free siRNA. The loading efficiency of siRNA was calculated by comparing the amount of siRNA that was not present in the supernatant to the amount of total siRNA.

Gel retardation assay. The binding of siRNA to chitosan was determined by electrophoresis using a 4\% agarose gel (low melting point). Nanoparticles with various chitosan/siRNA weight ratios were loaded onto the gel and subjected to electrophoresis. siRNA was visualized by ultraviolet light.

Serum stability assay. Chitosan/siRNA nanoparticles $(\sim 5 \mu \mathrm{g}$ siRNA, $200 \mu \mathrm{l}$ ) were incubated with an equal volume of $20 \%$ fetal bovine serum (FBS) in Dulbecco's modified Eagle's medium (DMEM) at $37^{\circ} \mathrm{C}$. At various time points $(0,0.5,2,4$, 7, 24, 48 and $72 \mathrm{~h}$ ), $30 \mu \mathrm{l}$ mixture was saved and stored at $-20^{\circ} \mathrm{C}$.

Characterization of the biological activity of chitosan/siRNA nanoparticles. CaSki cells were seeded in 96-well plates at a density of $3 \times 10^{4}$ cells/well and cultured in DMEM containing $10 \%$ FBS (no antibiotics) for $24 \mathrm{~h}$ prior to transfection. Chitosan/siRNA particles were added directly into the culture medium and the cells were cultured for an additional 24-48 h prior to examination by fluorescence microscopy.

Cell toxicity assay. Toxicity of chitosan was determined by the cell viability of chitosan/siRNA nanoparticles, as described previously (23).

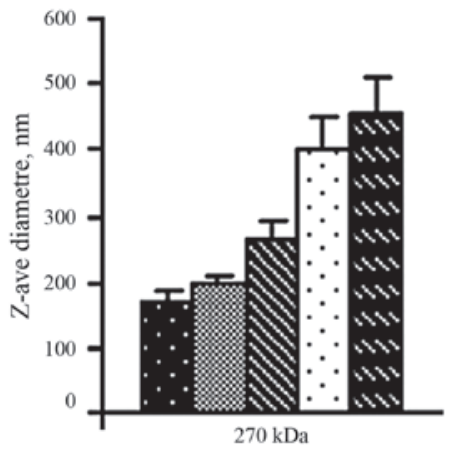

Figure 1. Characterisation of nanoparticles. Size of nanoparticles increasesd with the increasing weight ratio of chitosan to siRNA $(1.25: 1,2.5: 1,5: 1,10: 1$ and $15: 1)$

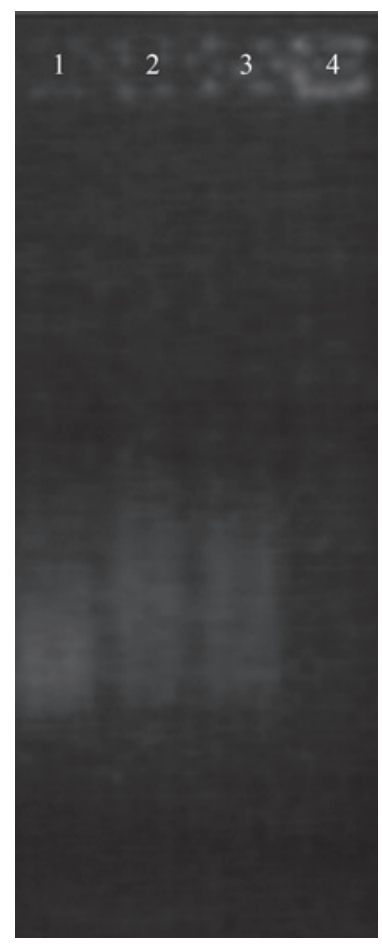

Figure 2. Gel retardation assay demonstrating siRNA in nanoparticles with various weight ratios of chitosan to siRNA (lanes 1-4 are 1.25:1, 2.5:1, 5:1, 10:1 and 15:1, respectively).

TUNEL staining. CaSki cells were seeded in 96-well plates at a density of $3 \times 10^{4}$ cells/well and cultured in DMEM containing $10 \% \mathrm{FBS}$ (no antibiotics) for $24 \mathrm{~h}$ prior to transfection. Chitosan/siRNA particles were added directly to the culture medium and the cells were cultured for an additional 24-48 h. Cell death was detected using an in situ Cell Death Detection kit (Nanjing KeyGen Biotech, Co., Ltd., Nanjing, China).

Western blot analysis. CaSki cells were seeded in 6-well plates at a density of $4 \times 10^{4}$ cells/well. Following plating ( $\left.24 \mathrm{~h}\right)$, cells were fed with fresh complete media and the chitosan/siRNA nanoparticles were added to the media. Following an additional $48 \mathrm{~h}$, cells were harvested with RIPA buffer. Samples were subjected to SDS-PAGE and immunoblotted with antibodies against HPV16 E7 and $\beta$-actin (Santa Cruz Biotechnology, Inc., Santa Cruz, CA, USA). 


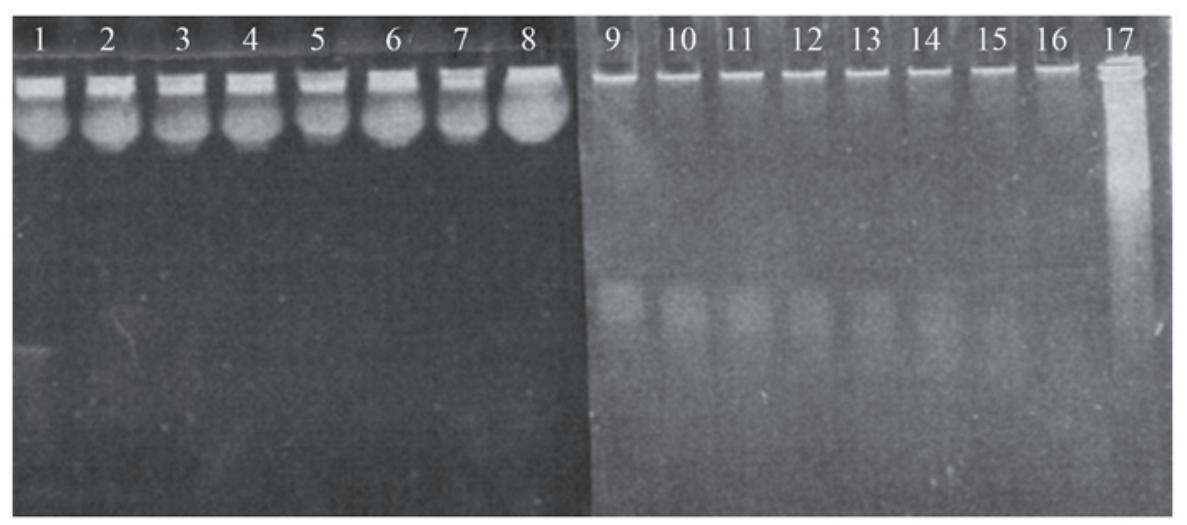

Figure 3. Degradation of naked siRNA and chitosan-binding siRNA in 20\% FBS-containing media at various time points (lanes 1-8 and lanes 9-17 are 0, 0.5, $1,2,4,7,24,48$ and $72 \mathrm{~h}$, respectively). Naked siRNA is completely degradated within $30 \mathrm{~min}$, whereas siRNA in chitosan/siRNA particles remains after 72-h incubation. FBS, fetal bovine serum.

A

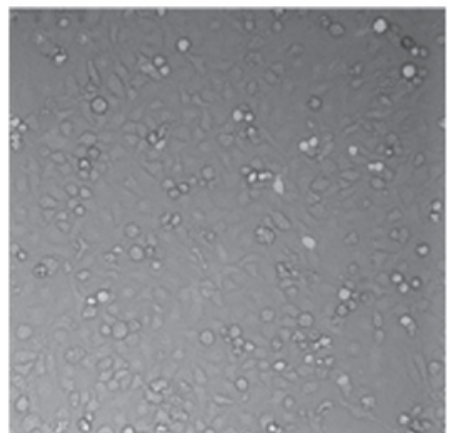

B

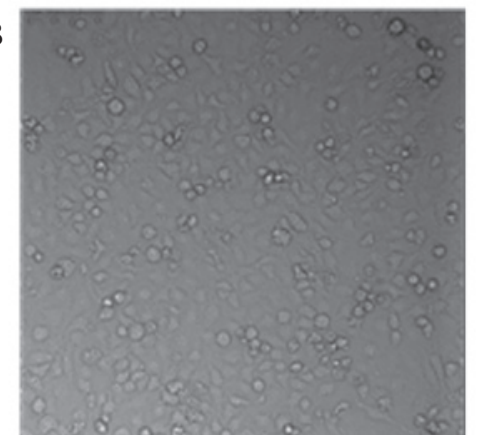

C

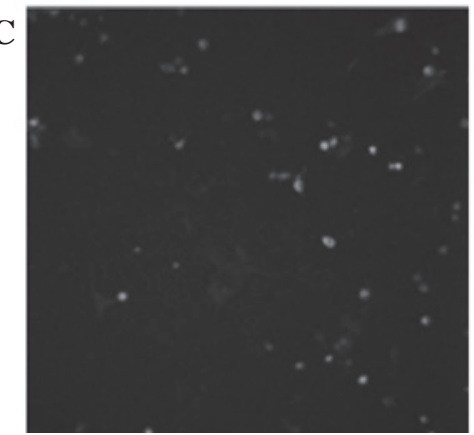

Figure 4. Images demonstrating the uptake of fluorescence-labeled siRNA/chitosan complexes by the cells. (A) $24 \mathrm{~h}$ following incubation with fluorescence-labeled siRNA/chitosan nanoparticles, (B) $24 \mathrm{~h}$ following incubation with non-labeled siRNA/chitosan nanoparticles, (C) $48 \mathrm{~h}$ following incubation with fluorescence-labeled siRNA/chitosan nanoparticles.

Table I. Alterations in $\zeta$ potential of nanoparticles with varied weight ratio of chitosan to siRNA.

\begin{tabular}{lc}
\hline $\begin{array}{l}\text { Chitosan } \\
\text { concentration }(\mu \mathrm{g} / \mathrm{ml})\end{array}$ & $\begin{array}{c}\zeta \text { potential } \\
(\mathrm{mV})\end{array}$ \\
\hline 25 & -11 \\
50 & -0.8 \\
100 & 51 \\
200 & 54 \\
300 & 55 \\
\hline
\end{tabular}

Amount of siRNA remained constant.

Statistical analysis. Data were analyzed using SPSS 11.0 (SPSS Inc., Chicago, IL, USA) and expressed as mean \pm SE. P $<0.05$ was considered to indicate a statistically significant difference.

\section{Results}

Size of chitosan/siRNA nanoparticles. The chitosan/siRNA particles formed by simple complexation had a diameter between 185 and $465 \mathrm{~nm}$ and the size increased with the increasing weight ratio of chitosan to siRNA (Fig. 1).
Surface charge. As revealed in Table I, the surface charge of chitosan/siRNA particles increased with increasing chitosan concentration (the amount of siRNA remained constant). Increased chitosan concentration increased the positive charge of the particles, preventing aggregation of the particles and enhancing their interaction with negatively charged cell membranes.

Interaction of siRNA with chitosan. Since chitosan and siRNA carry opposite charges, they are attracted to one another in solutions with specific $\mathrm{pH}$ values. Complete attachment of siRNA to chitosan was observed when chitosan and siRNA were mixed at a weight ratio of 100:1 (Fig. 2). The loading efficiency of siRNA was $72 \pm 1.5 \%$.

Stability of siRNA in serum. Naked siRNA was not stable in serum and was susceptible to enzyme digestion. When complexed with chitosan, the rate of degradation was markedly reduced (Fig. 3), indicating that chitosan protects siRNA from nuclease attack.

Biological activity of chitosan/siRNA nanoparticles. To examine the transfection efficiency of chitosan/siRNA nanoparticles, chitosan was complexed with fluorescence-labeled HPV16 E7 siRNA and their accumulation in CaSki cells was monitored. As demonstrated in Fig. 4, chitosan/siRNA particles 


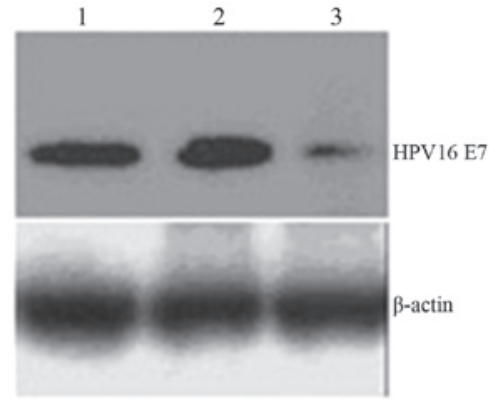

Figure 5. Western blot analysis demonstrating the effect of chitosan/siRNA on HPV16 E7 expression. Lane 1, chitosan alone; lane 2, chitosan/mock siRNA; lane 3, chitosan/HPV16 E7 siRNA. HPV, human papillomavirus.

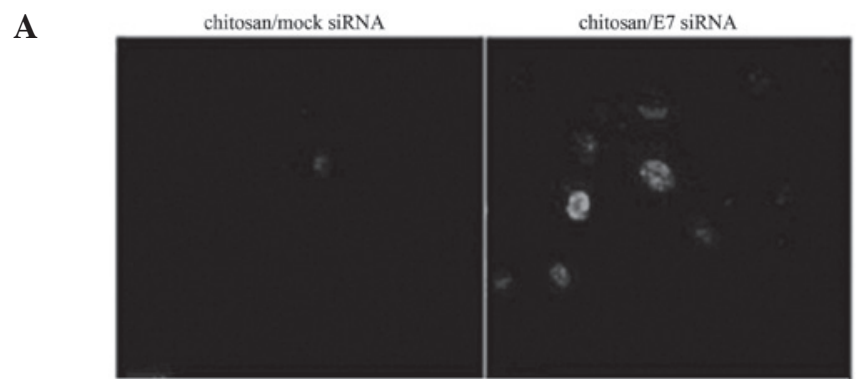

B

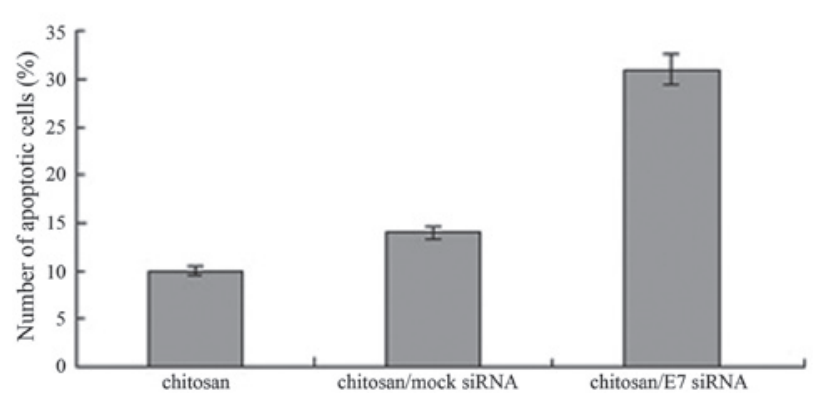

Figure 6. Effect of chitosan/siRNA on the induction of apoptosis in CaSki cells. (A) TUNEL staining demonstrating apoptotic cells incubated with chitosan/mock siRNA and chitosan/E7 siRNA. (B) Quantification of the number of apoptotic cells in cells treated with chitosan, chitosan/mock siRNA and chitosan/E7 siRNA.

were efficiently tranfected into cells following 24-h incubation. Protein levels of HPV16 E7 in CaSki cells were analyzed by western blot analysis and identified to be significantly downregulated (Fig. 5; P<0.05), indicating that chitosan/HPV16 E7 nanoparticles suppress expression of HPV16 E7.

Induction of apoptosis in CaSki cells by chitosan/HPV16 E7 nanoparticles. To examine the effect of chitosan/HPV16 E7 nanoparticles on cell apoptosis, apoptotic cells were detected using the TUNEL assay in cells treated with chitosan/HPV16E7 nanoparticles. A significantly higher number of apoptotic cells were detected in cells treated with chitosan/HPV16 E7 nanoparticles compared with cells treated with chitosan/mock siRNA particles (Fig. 6; $\mathrm{P}<0.05$ ).

\section{Discussion}

In the present study, chitosan/siRNA nanoparticles were prepared by simple complexation (24). The size and shape of nanoparticles is critical for efficient transfection of mammalian cells and distribution of nanoparticles in living cells (25). Previous studies have reported that nanoparticles exhibit higher levels of intracellular uptake compared with microparticles (26-28). This property is crucial for gene transfer, since the uptake of chitosan/DNA nanoparticles and their release from lysosomes are rate-limiting steps in this process $(29,30)$. Similar to DNA and oligodeoxyribonucleotides, siRNA is also taken up by cells (31). However, RNAi is not induced if siRNA fails to reach the cytoplasm (31). Using a carrier aids siRNA transfer into the intracellular compartment and protects it from enzyme degradation in lysosomes, thus efficiently inducing RNAi. In the present study, chitosan/siRNA nanoparticles were prepared with a size $<500 \mathrm{~nm}$ in diameter which were easily taken up by cells. The diameter of the nanoparticles increases with the increasing weight ratio of chitosan to siRNA. Therefore, nanoparticles of suitable sizes were prepared by adjusting the weight ratio of chitosan to siRNA.

Binding of siRNA to chitosan was demonstrated by gel retardation assay. The retarded migration of siRNA in agarose gel revealed binding of siRNA to chitosan. However, this binding is not as tight as that of DNA to chitosan, since DNA, but not siRNA, is concentrated by low concentration chitosan $(25 \mu \mathrm{g} / \mathrm{ml})$, indicating that siRNA binds to chitosan in a different manner to that of DNA to chitosan. Previously, the size of chitosan/DNA nanoparticles following concentration was reported to be 1,000 times smaller than that without concentration $(32,33)$ and the minimal size of DNA for concentration was 800 bp (32,34-36). In contrast to DNA, linearized siRNA is much shorter (21 bp). This property may account for the weak interaction of siRNA with chitosan. Since the size of nanoparticles remains unchanged following complexation, multiple, but not single siRNA may complex with chitosan.

The major cause of cervical cancer is infection with high-risk HPV. The integration of viral DNA into human genomes leads to the constitutive expression of oncoproteins E6 and E7, altering the cell cycle, immortalizing cells and causing cancer. Therefore, suppression of E7 expression may reverse the transformation process and induce apoptosis or senescence. Chitosan/HPV16 E7 siRNA nanoparticles were efficiently transfected into the cells (Fig. 4) and were found to suppress HPV16 E7 expression (Fig. 5). In addition, TUNEL staining revealed that apoptosis was induced in the CaSki cells. These results are consistent with previous studies. Chang et al (37) demonstrated that siRNA-mediated suppression of HPV E6 and E7 inhibited the growth of tumor cells from cervical cancer. Sima et al (38) reported that HPV16 E7 shRNA inhibits E6 and E7 expression and induces apoptosis in cancer cells via activation of p53, p21 and Rb. More recently, Guo et al (23) screened a phage display peptide library, identifying a heptapeptide which promotes degradation of E7 and prevent formation of E7/pRb complexes. This peptide induced $G_{1}$ phase arrest by restoration of $p R b$ activity, reinstating its ability to inhibit E2F activity. In addition, downregulation of E7 was reported to increase levels of p53 and induce apoptosis. Results of the current and previous studies indicate that suppression of HPV16 E7 by chitosan/siRNA nanoparticles inhibits growth of tumor cells and induces their apoptosis, which may serve as a potential therapy for cervical cancer. 


\section{References}

1. Ma B, Roden R and Wu TC: Current status of human papillomavirus vaccines. J Formos Med Assoc 109: 481-483, 2010.

2. Shao J, Chen ZY, Wang J and Ma R: Quantification of human papilloma virus DNA in the plasma of patients with cervical cancer. Clin J Surg Oncol 2: 288-291, 2010.

3. Qiao YL,Zhang WH, Li L, et al: The cross-sectional comparative research of cervical cancer gene screening method. Acta Acad Med Sin 24: 50-53, 2002.

4. Liu X, Roberts J, Dakic A, Zhang Y and Scheleqel R: HPV E7 contributes to the telomerase activity of immortalized and tumorigenic cells and augments E6-induced hTERT promoter function. Virology 375: 611-623, 2008.

5. Chu NR, Wu HB, Wu T, Boux LJ, Sieqel MI and Mizzen LA: Immunotherapy of a human papillomavirus (HPV) type 16 E7-expressing tumour by administration of fusion protein comprising mycobacterium bovis bacille calmette-guerin (BCG) hsp65 and HPV16 E7. Clin Exp Immunol 121: 216-225 2000.

6. Kaufmann AM, Stern PL, Rankin EM, et al: Safety and immunogenicity of TA-HPV, a recombinant vaccinia virus expressing modified human papillomavirus (HPV)-16 and HPV-18 E6 and E7 genes, in women with progressive cervical cancer. Clin Cancer Res 8: 3676-3685, 2002.

7. Veldman T, Horikawa I, Barrett JC and Schleqel R: Transcriptional activation of the telomerase hTERT gene by human papillomavirus type 16 E6 oncoprotein. J Virol 75: 4467-4472, 2001.

8. Nishimura A, Nakahara T, Ueno T, et al: Requirement of E7 oncoprotein for viability of HeLa cells. Microbes Infect 8: 984-993, 2006.

9. Dyson N, Howley PM, Münger K and Harlow E: The human papilloma virus-16 E7 oncoprotein is able to bind to the retinoblastoma gene product. Science 243: 934-937, 1989.

10. Boyer SN, Wazer DE and Band V: E7: protein of human papilloma virus-16 induces degradation of retinoblastoma protein through the ubiquitin-proteasome pathway. Cancer Res 56: 4620-4624, 1996.

11. Gonzalez SL, Stremlau M, He X, Basile JR and Münqer K: Degradation of the retinoblastoma tumor suppressor by the human papillomavirus type $16 \mathrm{E} 7$ oncoprotein is important for functional inactivation and is separable from proteasomal degradation of E7. J Virol 75: 7583-7591, 2001.

12. Gammoh N, Grm HS, Massimi P and Banks L: Regulation of human papillomavirus type 16 E7 activity through direct protein interaction with the E2 transcriptional activator. J Virol 80: 1787-1797, 2006.

13. Zhang B, Chen W and Roman A: The E7 proteins of low- and high-risk human papillomaviruses share the ability to target the pRB family member p130 for degradation. Proc Natl Acad Sci USA 103: 437-442, 2006.

14. Caldeira S, Dong W and Tommasino M: Analysis of E7/Rb associations. Methods Mol Med 119: 363-379, 2005.

15. Wu EW, Clemens KE, Heck DV and Münger K: The human papillomavirus E7 oncoprotein and the cellular transcription factor E2F bind to separate sites on the retinoblastoma tumor suppressor protein. J Virol 67: 2402-2407, 1993.

16. Alani RM and Münger K: Human papillomaviruses and associated malignancies. J Clin Oncol 116: 330-337, 1998.

17. Cobrinik D: Pocket proteins and cell cycle control. Oncogene 24: 2796-2809, 2005.

18. Dimova DK and Dyson NJ: The E2F transcriptional network: old acquaintances with new faces. Oncogene 24: 2810-2826, 2005 .
19. Helt AM and Galloway DA: Destabilization of the retinoblastoma tumor suppressor by human papillomavirus type 16 E7 is not sufficient to overcome cell cycle arrest in human keratinocytes. J Virol 75: 6737-6747, 2001.

20. Wang J, Sampath A, Raychaudhuri P and Baqchi S: Both Rb and E7 are regulated by the ubiquitin proteasome pathway in HPV-containing cervical tumor cells. Oncogene 20: 4740-4749, 2001.

21. Chen M, Gao S, Dong M, et al: Chitosan/siRNA nanoparticles encapsulated in PLGA nanofibers for siRNA delivery. ACS Nano 6: 4835-4844, 2012.

22. Holzerny P, Ajdini B, Heusermann W, Bruno K, Schuleit M, Meinel L and Keller M: Biophysical properties of chitosan/ siRNA polyplexes: profiling the polymer/siRNA interactions and bioactivity. J Control Release 157: 297-304, 2012.

23. Guo C, Liu K, Zheng Y, Luo H, Chen H and Huang L: Apoptosis induced by an antagonist peptide against HPV16 E7 in vitro and in vivo via restoration of p53. Apoptosis 16: 606-618, 2011.

24. Shu XZ and Zhu KJ: The influence of multivalent phosphate structure on the properties of ionically cross-linked chitosan films for controlled drug release. Eur J Pharm Biopharm 54: 235-243, 2002.

25. Gref R, Domb A, Quellec P, Blunkc T, Müllerd RH, Verbavatze JM and Langerf R: The controlled intravenous delivery of drugs using PEG-coated sterically stabilized nanospheres. Adv Drug Deliv Rev 16: 215-233, 1995.

26. Bivas-Benita M, Romeijn S, Junginger HE and Borchard G: PLGA-PEI nanoparticles for gene delivery to pulmonary epithelium. Eur J Pharm Biopharm 58: 1-6, 2004.

27. Panyam J and Labhasetwar V: Biodegradable nanoparticles for drug and gene delivery to cells and tissue. Adv Drug Deliv Rev 55: 329-347, 2003.

28. Zauner W, Farrow NA and Haines AM: In vitro uptake of polystyrene microspheres: effect of particle size, cell line and cell density. J Control Release 71: 39-51, 2001.

29. Huang M, Fong CW, Khor E and Lim LY: Transfection efficiency of chitosan vectors: effect of polymer molecular weight and degree of deacetylation. J Control Release 106: 391-406, 2005.

30. Chan V, Mao HQ and Leong KW: Effect of chitosan molecular weight in gene delivery process. Abs Pap Am Chem Soc 221: U347-U347, 2001.

31. Rozema DB and Lewis DL: siRNA delivery technologies for mammalian systems. Targets 2: 253-260, 2003.

32. Keller M: Lipidic carriers of RNA/DNA oligonucleotides and polynucleotides: what a difference a formulation makes. J Control Release 103: 537-540, 2005.

33. Tam P, Monck M, Lee D, et al: Stabilized plasmid-lipid particles for systemic gene therapy. Gene Ther 7: 1867-1874, 2000.

34. Bloomfield VA, He S, Li AZ and Arscott PB: Light scattering studies on DNA condensation. Biochem Soc Trans 19: 496, 1991.

35. Bloomfield VA: Condensation of DNA by multivalent cations: considerations on mechanism. Biopolymers 31: 1471-1481, 1991.

36. Bloomfield VA: DNA condensation. Curr Opin Struct Biol 6: 334-341, 1996.

37. Chang JT, Kuo TF, Chen YJ, et al: Highly potent and specific siRNAs against E6 or E7 genes of HPV16- or HPV18-infected cervical cancers. Cancer Gene Ther 17: 827-836, 2010.

38. Sima N, Wang W, Kong D, et al: RNA interference against HPV16 E7 oncogene leads to viral E6 and E7 suppression in cervical cancer cells and apoptosis via upregulation of $\mathrm{Rb}$ and p53. Apoptosis 13: 273-281, 2008. 